\title{
Effects of wild Thymus vulgaris essential oil on clinical isolates biofilm-forming bacteria
}

\author{
Jehad Al-Shuneigat ${ }^{*}$, Sameeh Al-Sarayreh ${ }^{1}$, Yousef Al-Saraireh ${ }^{1}$, Mahmoud \\ Al-Qudah ${ }^{2}$, Ibrahim Al-Tarawneh ${ }^{3}$ \\ I (Faculty of Medicine, Mutah University, Mutah, Jordan) \\ ${ }^{2}$ (Faculty of Science, Department of Chemistry Yarmouk University, Irbid, Jordan) \\ ${ }_{3}^{3}$ (Faculty of Science, Department of Chemistry, Albalqa' Applied university, Albalqa, Al-Salt, Jordan)
}

\begin{abstract}
:
Background: A biofilm is group of bacteria embedded in a self-produced matrix made of polysaccharide and protein. Bacterial biofilms cause chronic infections because they show increased tolerance to antibiotics and disinfectant chemicals as well as resisting phagocytosis. Biofilm bacteria show much greater resistance to antibiotics than their free-living counterparts. Essential oils are a cheap and effective alternative to antibiotics that could be used to combat antibiotics resistant microorganisms. The aim of the present study was to determine the effects of essential oil of wild Thymus vulgaris grown in Jordan on biofilm-forming clinical bacterial isolates.

Results: All of the isolates included in this study were able to produce a stable biofilm that adhered to polystyrene microwells. Thymus vulgaris essential oil produced inhibitory effects against all isolates with considerable variation in susceptibility. The minimum inhibitory concentration (MIC) and biofilm inhibitory concentration (BIC) values varied between 0.0625 to $2 \% \mathrm{v} / \mathrm{v}$. Isolate 6 (Pseudomonas aeruginosa) was the most resistant for both planktonic and biofilm growth while isolate 4 Escherichia coli was the most sensitive. Isolate 6 was the only isolate to exhibit a specific biofilm-related response to Thymus vulgaris essential oil i.e. the minimum Thymus vulgaris essential oil concentration needed to inhibit biofilm adherence was much lower than that required to inhibit planktonic growth. For the remaining isolates, BIC for the biofilm did not differ from the MIC for planktonic. Thymus vulgaris essential oil was able to inhibit Pseudomonas aeruginosa adherence to polystyrene.

Conclusions The MIC and BIC values of Thymus vulgaris essential oil show that the essential oil has strong antibacterial and antibiofilm activity. Thymus vulgaris was able to inhibit biofilm formation in the most tolerant isolate at sub-inhibitory concentrations.
\end{abstract}

Keywords: adherence, antibiotic resistance, biofilm, essential oil, MIC, BIC, Thymus vulgaris

\section{Introduction}

A biofilm, also known as exopolysaccharide, glycocalyx, or slime, is a population of cells that grows on the surface and embeds in a thick polysaccharide layer. It is believed that $99 \%$ of bacteria in nature exist in a biofilm $[1,2]$. Biofilm formation is a very important step in the pathogenicity of bacteria as biofilm microbes have become more resistant to antibiotics as well as to the immune system [3]. Biofilm provides protection for the bacteria against antimicrobials and other living cells so that cells in biofilm are about 1000 times more resistant to antimicrobial agents $[4,5]$. It is believed that about $60 \%$ of microbial infections are caused by biofilms [6]. The most common infections caused by biofilms are urinary tract infections caused by E. coli, infection related to catheter use by Staphylococcus epidermidis, middle-ear infections in children by Haemophilus influenzae, tooth decay by streptococci, burns and wound infections as well as lung infection by Pseudomonas aeruginosa [7], and Vibrio cholerae in the intestine [8].

In Germany alone more than 2.5 million biomedical devices are used including central venous catheters, prosthetic joints, cardiac pacemakers and heart valves, artificial lenses, and CSF-shunts. Each year billions of dollars are spent to replace biofilm infected implant devices with the infection rate in catheters reaching $100 \%[9]$.

The microbial resistance to antibiotics coupled with side effects of existing drugs increase the need for new drugs that are structurally and functionally different from currently used ones. The current trend is turning to nature" [10]. Many research institutes are examining and exploring nature in search of a novel antimicrobial with fewer side effects. There is mounting international literature supporting the use of plant-derived essential oils against pathogenic microorganisms [11]. Both clinical and in vitro studies have demonstrated the potent bactericidal properties of some essential oils, including efficacy against antibiotic-resistant strains such as MRSA [12]. The essential oils showed high penetrability and were more effective on organized biofilm than 
antibiotics [13]. Al-shuneigat et al 2005, demonstrated the antimicrobial effects of Polytoxinol ${ }^{\mathrm{TM}}$, a topical essential oil-based formulation; it was found to be able to inhibit biofilm formation of positive strains of coagulase-negative staphylococci in the most tolerant isolate at sub-inhibitory concentrations. Essential oils have been shown to interfere with bacterial colonization and the formation of biofilm on surfaces [14]. In addition essential oil stopped bacteria from aggregating and slowed their multiplication. This can lead to a reduced bacterial load, slow plaque maturation, and decreased plaque mass and pathogenicity. The bactericidal mechanisms of essential oils involve the rupture of the cell wall and enzymatic inhibition [13]. Thymus species have been shown to have strong antibacterial, antifungal, antiviral, antiparasitic, spasmolytic and antioxidant activities [15].

The aim of the present study was to determine the effects of essential oil of wild Thymus vulgaris $L$. type grown in Jordan on biofilm-forming clinical isolates bacteria.

\subsection{Essential oil of Thymus vulgaris}

\section{Materials And Methods}

In a previously published work [16] the composition of the essential oil from Thymus vulgaris grown in Jordan was determined using gas chromatography-mass spectrometry (GC-MS). Forty-eight components of the oil were identified representing more than $97 \%$ of the oil contents, monoterpenes being most abundant (about $85 \%$ ) with thymol (37.05\%), cis-dihydrocarvone $(9.34 \%)$, carvacrol $(8.45 \%)$, hydroxy-3-(3-methyl-2-butenyl)3 -cyclopenten-1-one $(8.41 \%)$, p-cymene $(5.73 \%)$, cis-sabinene $(4.42 \%)$, z-isoeugenol $(3.342 \%)$, and aromadendrene $(3.42 \%)$ as major constituents.

\subsection{Cultures and media}

The effect of Thymus vulgaris essential oil on bacterial biofilm formation was examined using 7 clinical isolates bacteria including: Methicillin-resistant Staphylococcus aureus (MRSA), Methicillin-sensitive Staphylococcus aureus (MSSA), Staphylococcus epidermidis, Escherichia coli, Klebsiella pneumonia, Pseudomonas aeruginosa and Proteus mirabilis. Cultures were stored on tryptone soya agar (TSA) (Oxoid, Hampshire, UK) at $2-4^{\circ} \mathrm{C}$ and subcultured every 2 months or whenever required. Isolates were purified on specific nutrient agar plates and characterized by standard microbiological and biochemical methods like Gram stain, catalase test, coagulase test and an API system (bioMerieux, France).

\subsection{Biofilm formation and broth microdilution assays}

Biofilm formation was quantified in microtitre plates using the method described by Rachid et al [17]. Bacteria were grown overnight in $10 \mathrm{ml}$ tryptone soya broth (TSB) at $37^{\circ} \mathrm{C}$ and then diluted $1: 100$ in fresh TSB supplemented with $0.5 \%$ glucose. For each test strain, $200 \mu \mathrm{l}$ of inoculum was added to 72 wells of a 96well plate. A quantity of $200 \mu \mathrm{l} \mathrm{TSB}$ was added to the remaining 24 wells and the plate incubated for $24 \mathrm{~h}$ at $37^{\circ} \mathrm{C}$. Following this the optical density at $600 \mathrm{~nm}\left(\mathrm{OD}_{600}\right)$ was measured as an indication of bacterial growth, the plate contents emptied out and washed three times with phosphate-buffered saline (PBS). The plates were air-dried and the cells that remained adhered to microwells stained with $0.4 \%$ crystal violet. $\mathrm{OD}_{490} \mathrm{~nm}$ was measured to quantify the amount of crystal violet-stained biofilm. Each strain was assayed in triplicate.

\subsection{BIC and MIC assays}

The BIC and MIC assays were performed in 96-well plates using a two fold dilution series, with each Thymus vulgaris essential oil concentration replicated eight times per plate. Planktonic growth and biofilm adherence were quantified as described above. Thymus vulgaris essential oil was used as a liquid formulation and concentrations are expressed as (\% v/v). MIC and BIC values were arbitrarily assigned as the minimum concentration that caused a 30\% decrease in optical density. MIC and BIC values assigned in this way for each dilution series within plates never varied by more than a single dilution step. The MIC and BIC value for each plate was taken to be the concentration that occurred most frequently. In the event of an equal split the MIC or BIC was taken as the higher concentration. Assays were performed three times on different days and for individual strains the same result was obtained on each occasion.

\subsection{Adherence of bacterial cells to polystyrene}

Initial adherence of bacterial cells to polystyrene was determined using a previously reported method [18]. Briefly, bacteria were grown overnight in $10 \mathrm{ml} \mathrm{TSB}$ at $37^{\circ} \mathrm{C}$ and then diluted $1: 100$ in fresh TSB containing Thymus vulgaris essential oil at the required concentration. A quantity of $5 \mathrm{ml}$ of the bacterial suspensions were then poured into Petri dishes and incubated for $30 \mathrm{~min}$ at $37^{\circ} \mathrm{C}$. The plates were washed five times using $5 \mathrm{ml}$ PBS, air dried and stained for $1 \mathrm{~min}$ with $0.4 \%$ crystal violet. The number of adhered cells was determined microscopically by counting the number of bacteria in 20 fields of view. Adherence was calculated 
as the total number of cells adhered per square centimetre examined. Each Thymus vulgaris essential oil concentration was assayed in triplicate and the adherence of Thymus vulgaris essential oil treated cells were compared with untreated controls.

\subsection{Quantification of biofilm formation}

\section{Results}

All isolates included in this study were able to produce a stable biofilm that adhered to polystyrene microwells (Fig. 1).

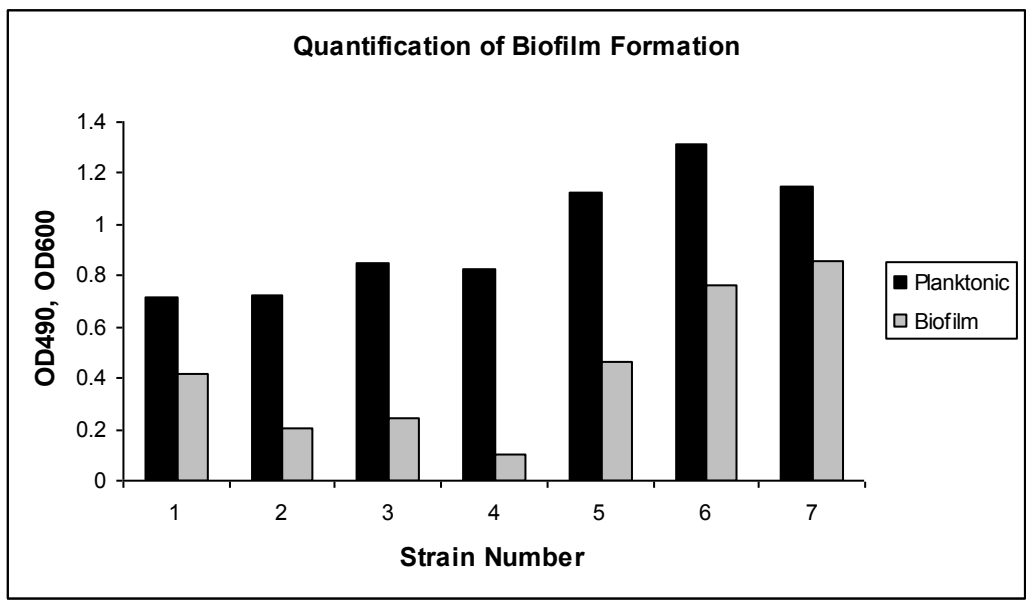

Figure 1. Quantification of Biofilm Formation

The optical density 600 was used to measure the planktonic growth while OD490 was used to measure biofilm growth. According to the amount of biofilm produced, the strains were classified into three groups: strains number 6 and 7 had an optical density $\left(\mathrm{OD}_{490}\right)$ of 0.854 and 0.762 respectively and were quantified as strong biofilm producers. Strains 1,5 , and 6 had an $\mathrm{OD}_{490}$ of more than 0.4 and were classified as moderate biofilm producers. Strains 2, 3 and 4 had an $\mathrm{OD}_{490}$ less than 0.4 and were classified as poor biofilm producers.

\subsection{Inhibition of bacterial growth by Thymus vulgaris, MIC and BIC values}

Thymus vulgaris essential oil produced inhibitory effects against all isolates (Table 1) with considerable variation in susceptibility. The MIC values as shown in Table 1 were between $0.0625-2 \% \mathrm{v} / \mathrm{v}$. Isolate 6 Pseudomonas aeruginosa was the most resistant for both planktonic and biofilm growth while isolate 4 Escherichia coli was the most sensitive.

Table 1. MIC of Thymus vulgaris for the clinical isolates used in this study

\begin{tabular}{|l|l|l|l|}
\hline Isolates number & \multicolumn{1}{|c|}{ MIC planktonic (\% v/v) } & \multicolumn{1}{|c|}{ BIC (\% v/v) } \\
\hline 1 & MRSA & 0.25 & 0.25 \\
\hline 2 & MSSA & 0.125 & 0.25 \\
\hline 3 & Staphylococcus epidermidis & 0.125 & 0.125 \\
\hline 4 & Escherichia coli & 0.0625 & 0.125 \\
\hline 5 & Klebsiella pneumonia & 0.125 & 0.25 \\
\hline 6 & Pseudomonas aeruginosa & 0.5 & 2 \\
\hline 7 & Proteus mirabilis & 0.25 & 0.25 \\
\hline
\end{tabular}

\subsection{Inhibition of Pseudomonas aeruginosa adherence to polystyrene by Thymus vulgaris at sub-MIC/BIC} levels

Adding sub-inhibitory concentrations of Thymus vulgaris essential oil to polystyrene Petri dishes containing a suspension culture of the Pseudomonas aeruginosa strain significantly reduced the number of individual cells adhering to the polystyrene surface after 30 minutes of incubation (Fig. 2). As shown in Fig 2, the higher the concentration of Thymus vulgaris essential oil, the lower was the number of bacteria adhered to polystyrene surface. Once the MIC/BIC value was reached the number of bacteria adhered significantly decreased. 


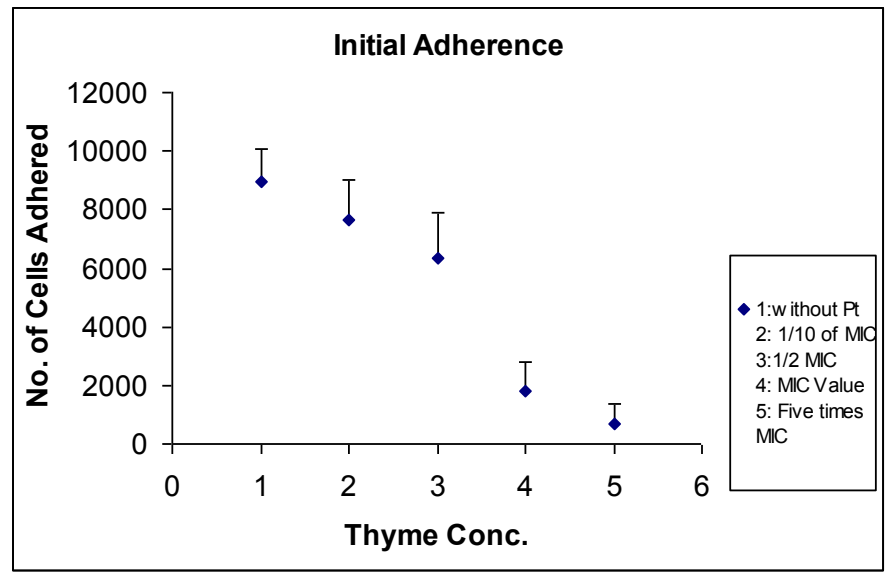

Figure 2. Effect of Thymus vulgaris on initial adhesion

\section{Discussion}

Thymus vulgaris essential oil has been used for centuries in traditional medicine. This study reports on the effects of wild Thymus vulgaris essential oil on clinical isolates biofilm-forming bacteria. A hallmark of biofilms is their profound tolerance against antimicrobial agents. A variety of reasons for the increased antimicrobial resistance of microorganisms in biofilms have been suggested. The extracellular matrix provides physically restriction to the diffusion of antimicrobial agents. In addition, nutrient and oxygen depletion within the biofilm cause some bacteria to enter stationary state, in which they are less susceptible to growth dependent antimicrobial killing. Besides, some organisms in biofilms have been shown to express biofilm-specific antimicrobial resistance genes.

The results of the present study showed that Thymus vulgaris essential oil has strong antibacterial and antibiofilm capacity. The MIC and BIC values were between $0.0625-2 \% \mathrm{v} / \mathrm{v}$ for all isolates. Isolate 6 (Pseudomonas aeruginosa) was the only isolate to exhibit a specific biofilm-related response to Thymus vulgaris essential oil i.e. the minimum Thymus vulgaris essential oil concentration that inhibited biofilm adherence was much lower than that required to inhibit planktonic growth. For the remaining isolates, BIC did not differ that much from the MIC.

The effectiveness of Thymus vulgaris essential oil may be correlated with the content of thymol $(37.05 \%)$, which is one of the most active constituents with a wide antimicrobial spectrum. The activity of thymol is attributed to the characteristic feature of the phenolic hydroxyl group that is more acidic than that of the aliphatic hydroxyl group due to the presence of delocalized electrons [19]. Carvacrol is another constitutes of Thymus vulgaris essential oil that makes about $8.45 \%$ of the oil and is very important for the antimicrobial activity of the essential oil. Carvacrol destabilizes the cytoplasmic membrane as it has the ability to form hydrogen bonds between hydroxyl group in these compounds and water molecules in bacterial cell and that will disables dynamic actions in bacterial cell [20]. Thymus vulgaris essential oil was able to inhibit Pseudomonas aeruginosa adherence to polystyrene at subinhibitory level (Fig 3). It is believed that the subinhibitory level of Thymus vulgaris essential oil that did not cause killing of the microorganism might have caused damage and changes to Pseudomonas aeruginosa cell membrane and prevented it from adhering to the polystyrene surface.
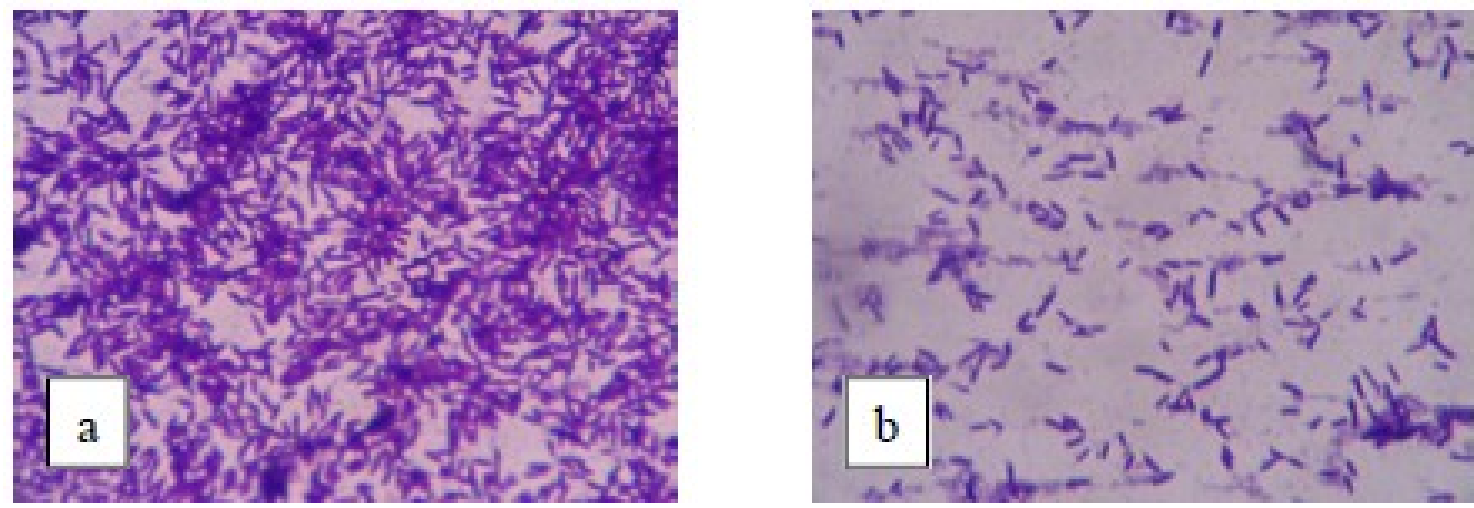

Figure 3. Microscopic images of Pseudomonas aeruginosa adherence to polystyrene a. without Thymus vulgaris essential oil b. with Thymus vulgaris essential oil. 


\section{Conclusion}

In conclusion, we have demonstrated the antimicrobial efficacy of Thymus vulgaris essential oil against seven clinical isolates bacteria in vitro. Thymus vulgaris essential oil produced inhibitory effects against all isolates at low concentrations. At sub-inhibitory levels Thymus vulgaris reduced the ability of Pseudomonas aeruginosa isolate to adhere to a polystyrene surface.

\section{References}

[1]. A. Stewart, Biofilm bacteria in chronic middle ear infection. Molecular Medicine Today, 141, 1998, 1357-4310.

[2]. R. Van Houdt, and C.W. Michiels, Biofilm formation and the food industry, a focus on the bacterial outer surface. Journal of Applied Microbiology, 109, 2010, 1117-1131

[3]. G. Reid, J. Howard, and B.S. Gan, Can bacteria interference prevent infection. Trends in Microbiology, 9(9), 2001, 424-428.

[4]. T. F. Mah, and G. A. O'Toole, Mechanisms of biofilm resistance to antimicrobial agents. Trends in Microbiology, 9(11), 2001, 34-9.

[5]. C. Fuente-Nunez, J. Mertens, J. Smit, and R. E. W Hancock, The bacterial surface layer provides protection against antimicrobial peptides. Applied and Environmental Microbiology, 78(15), 2012, 5452-5456.

[6]. K. Lewis, Riddle of Biofilm Resistance. Antimicrobial Agents and Chemotherapy, 45(4), 2001, 999-1007.

[7]. C. Stephens, Microbiology: Breaking down biofilms. Current Biology, 12, 2002, R132-R134.

[8]. P. Das, Paula I Watnick-elucidating the role of biofilms. The Lancet Infectious Diseases, 2, 2002, $190-192$.

[9]. D. Mack, P. Becker, I. Chatterjee, S. Dobinsky, J. Knobloch, G. Peters, H. Rohde, and M. Herrmann, Mechanisms of biofilm formation in Staphylococcus epidermidis and Staphylococcus aureus: functional molecules, regulatory circuits, and adaptive responses. International Journal of Medical Microbiology, 294 2004, 203-212.

[10]. Lawless J, Tea tree oil (United Kingdom, Thorsons, 1994).

[11]. E. Sherry, H. Boeck, and P. H. Warnke, Percutaneous treatment of chronic MSRA osteomyelitis with a novel plant-derived antiseptic. BMC Surgery, 1, 2001, 1-1.

[12]. M. Harkenthal, J. Reichling, H. K. Geiss, and R. Saller R, Comparative study on the in vitro antibacterial activity of Australian tea tree oil, cajuput oil, niaouli oil, manuka oil, kanuka oil, and eucalyptus oil. Pharmazie, 54(6), 1999, 460-463.

[13]. D. H. Fine, D. Furgang, R. Lieb, I. Korik, J. W. Vincent, and M. L. Barnett, Effects of sublethal exposure to an antiseptic mouthrinse on representative plaque bacteria. J Clin Periodontol, 23(15), 1996, 444-451.

[14]. J. Al-Shuneigat, S. D. Cox, and J. L. Markham, Effects of a topical essential oil-containing formulation on biofilm-forming coagulase-negative staphylococci. Letters in Applied Microbiology, 41(1), 2005, 52-55.

[15]. E. Stahl-Biskup, and F. Saez, Thyme (London, Taylor and Francis, 2002).

[16]. Al-Shuneigat. J, Al-Sarayreh. S, Al-Saraira Y, Al-Qudah. M, Al-Tarawneh I, Al-Dalaen. S, Chemical composition and antimicrobial activity of the essential oil of wild Thymus vulgaris grown in South Jordan. IOSR Journal of Pharmacy and Biological Sciences 9(5), 2014, 78-82.

[17]. S. Rachid, K. Ohlsen, W. Witte, J. Hacker, and W. Ziebuhr, Effect of Subinhibitory antibiotic concentrations on polysaccharide intercellular adhesion expression in biofilm-forming Staphylococcus epidermidis. Antimicrobial Agents and Chemotherapy, 44(12), 2000, 3357-3363.

[18]. C. Heilmann, C. Gerke, F. P. Premington, and F. Gotz, Characterization of Tn917 insertion mutant of Staphylococcus epidermidis affected in biofilm formation. Infection and Immunity, 64(1), 1996, 277-282.

[19]. A. Ultee, M. H. J. Bennik, and R. Moezelaar, The phenolic hydroxyl group of carvacrol is essential for action against the foodborne pathogen Bacillus cereus. Applied and Environmental Microbiology, 68, 2002, 1561-1568.

[20]. N. K. Fayad, O. H. S. AL- Obaidi, T. H. Al-Noor, and M. O. Ezzat, Water and alcohol extraction of thyme plant (Thymus vulgaris) and activity study against bacteria, tumors and used as anti-oxidant in margarine manufacture. Innovative Systems Design and Engineering, 4(1), 2013, 41-51. 